\title{
Factors that Needs Improvement in Participatory Budgeting Projects to Achieve Community Development Goals in Port Loko District and City Council
}

\author{
D.E.M. Lavalie ${ }^{*}$, S. Subadra ${ }^{2}$ and S. P. Sreekala ${ }^{3}$ \\ ${ }^{1}$ Research Scholar, School of Business Management \\ Department of Business Management, Texila American University \\ 2Department of Commerce, NKR Government Arts College for Women, Namakkal 637001 \\ ${ }^{3}$ Associate Professor, KPR arts and Science College, Coimbatore \\ E-mail: demlavalie@gmail.com; dr.subadraphd@gmail.com; spsreekalaashok@gmail.com \\ *Corresponding author details: Daniel E.M. Lavalie; demlavalie@gmail.com

\begin{abstract}
The purpose of the study is to find out and recommend the factors that need improvement in the future implementation of participatory budgeting in Port Loko District and City Council in order to achieve community development goals. As our study is based on primary data, we collected 420 questionnaires to analyse our findings. A lot of factors were identified and analysed using Garret Ranking Techniques.
\end{abstract}

Keywords: local council; primary data; port Loko district and city council; participatory budgeting; Garrett ranking test

\section{INTRODUCTION}

The functions devolved to local councils according to (Commonwealth Governance ) are primary and secondary health, primary and junior secondary educations, environmental health, agriculture extension services, rural water supply, waste management and community development. Again, in the Local Government (ACT, 2004) section 20 (1), local council is charged with the function of: A local council shall be the highest political authority in the locality and shall have legislative and executive powers to be exercised in accordance with this Act or any other enactment, and shall be responsible, generally for promoting the development of the locality and the welfare of the people in the locality with the resources at its disposal and with such resources and capacity as it can mobilise from the central government and its agencies, national and international organisations, and the private sector. (p.16)

These above functions delegated to councils do not go without problems and challenges ranging from late disbursement of funds, bureaucracy in the process, the Participatory Budgeting selection process, small own source revenue generation, to citizens' lack of trust in council for development project implementation. Participatory budgeting according to (LGA, 2020):

Participatory budgeting is a form of citizen participation in which citizens are involved in the process of deciding how public money is spent. Local people are often given a role in the scrutiny and monitoring of the process following the allocation of budgets.
Also, according to (EUKN), in Lisbon Participatory Budget, it defined participatory budgeting as:

\begin{abstract}
"a way of including the citizens in the decision-making process of the city budget. It can imply, in some cities, a whole administrative structure feeding on grassroots democracy, which defines budget priorities for the local neighbourhood or district. In other cases, the city allocates part of the budget to projects which are decided by the citizens: the citizens can suggest projects, and then they can vote in their favourite ones"
\end{abstract}

These and many other functions need continuous improvements for the councils to effectively and efficiently deliver their services and work in the development of their locality. The objective of the study is to identify factors that needs improvements and recommends such factors to be improved on if participatory budgeting programmes wants to achieve community development goals. To achieve the above objectives Garrett ranking techniques was used to analyse the respondents' views from the administered questionnaire.

\section{OBJECTIVES}

The objectives of the study are:

1) To find the factors that needs improvement for effective and efficient participatory budgeting in the Port Loko District and City Council.

2) To recommend on the factors that need improvement for efficient and effective Participatory budgeting in the Port Loko District and City Councils to achieve future programme implementation that is fully participatory in nature. 


\section{METHODOLOGY}

\section{Garrett Ranking Techniques}

This method of ranking was used to determine from the respondents the factors that needs improvements and recommends for such in the implementation of effective and efficient participatory budgeting in order to achieve community development goals.

According to (Rahman, 2011), Garrett technique is used by policy holders after ranking factors according to their magnitude. The order of the merit given by respondents was converted into ranks by using the formula below:

Formula

Position $=\quad \frac{100(\mathrm{Rij}-0.5) \text { Percentage }}{\mathrm{Nj}}$
Where: $\mathbf{R i j}=$ Rank given for the $\mathrm{i}^{\text {th }}$ variable by the $\mathbf{j}^{\text {th }}$ respondent

$\mathbf{N j}=$ Number of variables ranked by the $\mathrm{j}^{\text {th }}$ respondent.

This technique according to (Rahman, 2011) again:

The percentage position of each rank thus obtained was converted in to scores by referring to the table given by Henry Garrett. Then for each factor the scores of individual respondents were added together and divided by the total number of respondents for who the scores were added. These mean scores for all the factors were arranged in the order of their ranks and inferences were drawn

\section{Port Loko District and City Council}

The tables below show the ranks given to the factors to be improved on by Port Loko District and City Council if participatory budgeting wants to be fully effective and efficient for the achievement of community development goals in the future.

APPENDIX -B

TABLE 1: GARRETT'S RANKING TABLE

\begin{tabular}{|c|c|c|c|}
\hline PERCENTAGE & SCORE & PERCENTAGE & SCORE \\
\hline 0.09 & 99 & 52.02 & 49 \\
\hline 0.20 & 98 & 54.03 & 48 \\
\hline 0.32 & 97 & 56.03 & 47 \\
\hline 0.45 & 96 & 58.03 & 46 \\
\hline 0.61 & 95 & 59.99 & 45 \\
\hline 0.78 & 94 & 61.94 & 44 \\
\hline 0.97 & 93 & 63.85 & 43 \\
\hline 1.18 & 92 & 65.75 & 42 \\
\hline 1.42 & 91 & 67.48 & 41 \\
\hline 1.68 & 90 & 69.39 & 40 \\
\hline 1.96 & 89 & 71.14 & 39 \\
\hline 2.28 & 88 & 72.85 & 38 \\
\hline 2.63 & 87 & 74.52 & 37 \\
\hline 3.01 & 86 & 76.12 & 36 \\
\hline 3.43 & 85 & 77.68 & 35 \\
\hline 3.89 & 84 & 79.12 & 34 \\
\hline 4.38 & 83 & 80.61 & 33 \\
\hline 4.92 & 82 & 81.99 & 32 \\
\hline 5.51 & 81 & 83.31 & 31 \\
\hline 6.14 & 80 & 84.56 & 30 \\
\hline
\end{tabular}




\begin{tabular}{|c|c|c|c|}
\hline PERCENTAGE & SCORE & PERCENTAGE & SCORE \\
\hline 6.81 & 79 & 85.75 & 29 \\
\hline 7.55 & 78 & 86.89 & 28 \\
\hline 8.33 & 77 & 87.96 & 27 \\
\hline 9.17 & 76 & 88.97 & 26 \\
\hline 10.16 & 75 & 89.94 & 25 \\
\hline 11.03 & 74 & 90.83 & 24 \\
\hline 12.04 & 73 & 91.67 & 23 \\
\hline 13.11 & 72 & 92.45 & 22 \\
\hline 14.25 & 71 & 93.19 & 21 \\
\hline 15.44 & 70 & 93.86 & 20 \\
\hline 16.69 & 69 & 94.49 & 19 \\
\hline 18.01 & 68 & 95.08 & 18 \\
\hline 19.39 & 67 & 95.62 & 17 \\
\hline 20.93 & 66 & 96.11 & 16 \\
\hline 22.32 & 65 & 96.57 & 15 \\
\hline 23.88 & 64 & 96.99 & 14 \\
\hline 25.48 & 63 & 97.37 & 13 \\
\hline 27.15 & 62 & 98.72 & 12 \\
\hline 28.86 & 61 & 98.04 & 11 \\
\hline 30.61 & 60 & 98.32 & 10 \\
\hline 32.42 & 59 & 98.58 & 9 \\
\hline 34.25 & 58 & 99.82 & 8 \\
\hline 36.15 & 57 & 99.03 & 7 \\
\hline 38.06 & 56 & 99.22 & 6 \\
\hline 40.01 & 55 & 99.39 & 5 \\
\hline 41.97 & 54 & 99.55 & 4 \\
\hline 43.97 & 53 & 99.68 & 3 \\
\hline 45.97 & 52 & 99.80 & 2 \\
\hline 47.98 & 51 & 99.91 & 1 \\
\hline 50.00 & 50 & 100.00 & 0 \\
\hline
\end{tabular}

SOURCE: Henry, E. Garret’s, Statistics in Psychology and Education, Feffer and Simans Private Limited, 1969, p.329 


\section{FIRST SET OF FACTORS}

TABLE 2: Determinant of participatory budgeting Factors

\begin{tabular}{|c|c|c|c|c|c|}
\hline S. No & F1 & F2 & F3 & F4 & Total \\
\hline 1 & 49 & 205 & 98 & 68 & 420 \\
\hline 2 & 61 & 85 & 97 & 177 & 420 \\
\hline 3 & 78 & 61 & 196 & 85 & 420 \\
\hline 4 & 232 & 69 & 29 & 90 & 420 \\
\hline Total & 420 & 420 & 420 & 420 & 1680 \\
\hline
\end{tabular}

Source: Primary Data

TABLE 3: Percentage Position Determination

$\begin{array}{ccc}\text { Rank } & \text { Percentage }=100(\mathrm{Rij}-0.05) / \mathrm{Nj} & \text { Garret Score } \\ 1 & 12.5 & \mathbf{7 4} \\ 2 & 37.5 & 57 \\ 4 & 62.5 & 28\end{array}$

Source: Primary Data

TABLE 4: Garret Mean Score

\begin{tabular}{|c|c|c|c|c|c|c|c|c|}
\hline \multirow{2}{*}{ S. No } & \multicolumn{2}{|c|}{ F1 } & \multicolumn{2}{|c|}{ F2 } & \multicolumn{2}{|c|}{ F3 } & \multicolumn{2}{|c|}{ F4 } \\
\hline & Freq. & GMS & Freq. & GMS & Freq. & GMS & Freq. & GMS \\
\hline 1 & 49 & 8.633333 & 205 & 36.11905 & 98 & 17.26667 & 68 & 11.98095 \\
\hline 2 & 61 & 8.278571 & 85 & 11.53571 & 97 & 13.16429 & 177 & 24.02143 \\
\hline 3 & 78 & 8.171429 & 61 & 6.390476 & 196 & 20.53333 & 85 & 8.904762 \\
\hline 4 & 232 & 15.46667 & 69 & 4.6 & 29 & 1.933333 & 90 & 6 \\
\hline $\begin{array}{l}\text { Tota } \\
\text { Mea }\end{array}$ & $\begin{array}{l}\text { rret } \\
\text { core }\end{array}$ & 40.55 & & 58.64524 & & 52.89762 & & 50.90714 \\
\hline
\end{tabular}

Source: Primary Data

TABLE 5: Factor Determinant of Participatory Budgeting to be improved on

\begin{tabular}{|c|c|c|c|}
\hline Factor & Mean & Rank & \\
\hline 1 & $\mathbf{4 0 . 5 5}$ & 4 & Availability of fund \\
\hline 2 & $\mathbf{5 8 . 6 4 5}$ & 1 & Public participation \\
\hline 3 & $\mathbf{5 2 . 8 9 8}$ & 2 & Citizens decides how funds are spent \\
\hline 4 & $\mathbf{5 0 . 9 0 7}$ & 3 & Project implemented based on views of the voting public \\
\hline
\end{tabular}

Source: Primary Data 


\section{SECOND SET OF FACTORS}

TABLE 6: Awareness and Public Participation Factor to be improved on

\begin{tabular}{|c|c|c|c|c|c|c|}
\hline S. No & F1 & F2 & F3 & F4 & F5 & Total \\
\hline 1 & 294 & 84 & 42 & $\mathbf{0}$ & 0 & 420 \\
\hline 2 & 84 & 168 & 84 & 42 & 42 & 420 \\
\hline 3 & 0 & 84 & 168 & 84 & 84 & 420 \\
\hline 4 & $\mathbf{0}$ & 42 & 126 & 210 & 42 & 420 \\
\hline 5 & 42 & 42 & 0 & 84 & 252 & 420 \\
\hline Total & 420 & 420 & 420 & 420 & 420 & 2100 \\
\hline
\end{tabular}

Source: Primary Data

TABLE 7: Percentage Position Determination

\begin{tabular}{|c|c||c||}
\hline \hline Rank & Percentage=100(Rij-0.05)/Nj & Score \\
\hline 1 & 10 & 61 \\
3 & 30 & 50 \\
4 & 50 & 40 \\
5
\end{tabular}

Source: Primary Data

TABLE 8: Garrett Mean Score

\begin{tabular}{|c|c|c|c|c|c|c|c|c|c|c|}
\hline \multirow{2}{*}{ S. No } & \multicolumn{2}{|c|}{ F1 } & \multicolumn{2}{|c|}{ F2 } & \multicolumn{2}{|c|}{ F3 } & \multicolumn{2}{|c|}{ F4 } & \multicolumn{2}{|c|}{ F5 } \\
\hline & Freq. & GMS & Freq. & GMS & Freq. & GMS & Freq. & GMS & Freq. & GMS \\
\hline 1 & 294 & 53.2 & 84 & 15.2 & 42 & 7.6 & 0 & 0 & 0 & 0 \\
\hline 2 & 84 & 12.2 & 168 & 24.4 & 84 & 12.2 & 42 & 6.1 & 42 & 6.1 \\
\hline 3 & 0 & 0 & 84 & 10 & 168 & 20 & 84 & 10 & 84 & 10 \\
\hline 4 & 0 & 0 & 42 & 4 & 126 & 12 & 210 & 20 & 42 & 4 \\
\hline 5 & 42 & 2.5 & 42 & 2.5 & 0 & 0 & 84 & 5 & 252 & 15 \\
\hline $\begin{array}{l}\text { Total } \\
\text { GMS }\end{array}$ & & 67.9 & & 56.1 & & 51.8 & & 41.1 & & 35.1 \\
\hline
\end{tabular}

Source: Primary Data

\section{THIRD SET OF FACTORS}

TABLE 9: Awareness and public participation factor to be improved on from Garrett mean score ranking.

\begin{tabular}{|c|c|c|c|}
\hline FACTOR & MEAN & RANK & \\
\hline F1 & 67.9 & 1 & Just inform citizens \\
\hline F2 & 56.1 & 2 & Consult citizens \\
\hline F3 & 51.8 & 3 & Involve citizens \\
\hline F4 & 41.1 & 4 & Collaborate with citizens \\
\hline F5 & 35.1 & 5 & Empower citizens \\
\hline
\end{tabular}

Source: Primary Data 
TABLE 10: Stages of participatory budgeting factor to be improved on

\begin{tabular}{cccccc}
\hline S. No & F1 & F2 & F3 & F4 & Total \\
\hline \hline 1 & 258 & 84 & 0 & 78 & 420 \\
\hline 3 & 42 & 231 & 105 & 42 & 420 \\
\hline 4 & 0 & 63 & 294 & 21 & 420 \\
\hline \hline Total & 120 & 42 & 21 & 420 & 1680 \\
\hline \hline
\end{tabular}

Source: Primary Data

TABLE 11: Percentage Position Determination

\begin{tabular}{cccc} 
Rank & Percentage $=\mathbf{1 0 0}(\mathrm{Rij}-\mathbf{0 . 0 5}) / \mathrm{Nj}$ & Score \\
1 & 12.5 & 57 & 74 \\
2 & 37.5 & 44 \\
\hline & 62.5 & 28
\end{tabular}

Source: Primary Data

TABLE 12: Garrett Mean Score

\begin{tabular}{|c|c|c|c|c|c|c|c|c|}
\hline \multirow{2}{*}{ S. No } & \multicolumn{2}{|c|}{ F1 } & \multicolumn{2}{|c|}{ F2 } & \multicolumn{2}{|c|}{ F3 } & \multicolumn{2}{|c|}{ F4 } \\
\hline & Freq. & GMS & Freq. & GMS & Freq. & GMS & Freq. & GMS \\
\hline 1 & 258 & 45.45714 & 84 & 14.8 & $\mathbf{0}$ & $\mathbf{0}$ & 78 & 13.74286 \\
\hline 2 & 42 & 5.7 & 231 & 31.35 & 105 & 14.25 & 42 & 5.7 \\
\hline 3 & $\mathbf{0}$ & $\mathbf{0}$ & 63 & 6.6 & 294 & 30.8 & 63 & 6.6 \\
\hline 4 & 120 & 8 & 42 & 2.8 & 21 & 1.4 & 237 & 15.8 \\
\hline Total & & 59.15714 & & 55.55 & & 46.45 & & 41.84286 \\
\hline
\end{tabular}

Source: Primary Data

TABLE 13: Stages of participatory budgeting factor to be improved on based on Garrett mean score ranking

\begin{tabular}{|c|c|c|c|}
\hline Factor & Mean & Rank & \\
\hline F1 & 59.15714 & 1 & Identify needs \\
\hline F2 & 55.55 & 2 & Develop project proposal \\
\hline F3 & 46.45 & 3 & Select project to be funded \\
\hline F4 & 41.84286 & 4 & Implement, Monitoring, Evaluate and report \\
\hline
\end{tabular}

Source: Primary Data 


\section{FOURTH SET OF FACTORS}

TABLE 14: General areas of participatory budgeting to be improved on

\begin{tabular}{|c|c|c|c|c|c|c|}
\hline S. No & F1 & F2 & F3 & F4 & F5 & Total \\
\hline 1 & 200 & 135 & 0 & 85 & 0 & 420 \\
\hline 2 & 0 & 262 & 65 & 71 & 22 & 420 \\
\hline 3 & 21 & 0 & 74 & 226 & 99 & 420 \\
\hline 4 & 60 & 23 & 200 & 38 & 99 & 420 \\
\hline 5 & 139 & 0 & 81 & 0 & 200 & 420 \\
\hline Total & 420 & 420 & 420 & 420 & 420 & 2100 \\
\hline
\end{tabular}

Source: Primary Data

TABLE 15: Percentage Position Determination

\begin{tabular}{|c|c|c|}
\hline Rank & Percentage $=\mathbf{1 0 0}(\mathbf{R i j}-\mathbf{0 . 0 5}) / \mathbf{N j}$ & Score \\
\hline 1 & 10 & 61 \\
\hline 2 & 30 & 50 \\
\hline 4 & 50 & 40 \\
\hline 5 & 70 & 25 \\
\hline
\end{tabular}

Source: Primary Data

TABLE 16: Garrett Mean Score

\begin{tabular}{|c|c|c|c|c|c|c|c|c|c|c|}
\hline \multirow{2}{*}{ S. No } & \multicolumn{2}{|c|}{ F1 } & \multicolumn{2}{|c|}{ F2 } & \multicolumn{2}{|c|}{ F3 } & \multicolumn{2}{|c|}{ F4 } & \multicolumn{2}{|c|}{ F5 } \\
\hline & Freq. & GMS & Freq. & GMS & Freq. & GMS & Freq. & GMS & Freq. & GMS \\
\hline 1 & 200 & 36.19048 & 135 & 24.42857 & 0 & 0 & 85 & 15.38095 & 0 & 0 \\
\hline 2 & 0 & 0 & 262 & 38.05238 & 65 & 9.440476 & 71 & 10.3119 & 22 & 3.195238 \\
\hline 3 & 21 & 2.5 & 0 & 0 & 74 & 8.809524 & 226 & 26.90476 & 99 & 11.78571 \\
\hline 4 & 60 & 5.714286 & 23 & 2.190476 & 200 & 19.04762 & 38 & 3.619048 & 99 & 9.428571 \\
\hline 5 & 139 & 8.27381 & 0 & 0 & 81 & 4.821429 & 0 & 0 & 200 & 11.90476 \\
\hline Tot & MS & $\mathbf{5 2 . 6 7 8 5 7}$ & & 64.67143 & & 42.11905 & & 56.21667 & & 36.31429 \\
\hline
\end{tabular}

Source: Primary Data

TABLE 17: General areas of participatory budgeting to be improved on based on Garrett mean score

\begin{tabular}{|c|c|c|l|}
\hline FACTOR & MEAN & RANK & \\
\hline F1 & $\mathbf{5 2 . 6 7 8 5 7 1 4}$ & 3 & Provide feedback \\
\hline F2 & 64.6714286 & 1 & Projects selected are implemented \\
\hline F3 & 42.1190476 & 4 & Are always informed \\
\hline F4 & 56.2166667 & 2 & Inputs/recommendations implemented \\
\hline F5 & 36.3142857 & 5 & My ward benefited \\
\hline
\end{tabular}

Source: Primary Data 


\section{FIFTH SET OF FACTORS}

TABLE 18: Effectiveness factor to be improved on

\begin{tabular}{||c||c||c||c||c||c||c|}
\hline RANK & F1 & F2 & F3 & F4 & F5 & Total \\
1 & 11 & 45 & 24 & 25 & 315 & 420 \\
2 & 306 & 32 & 4 & 10 & 68 & 420 \\
3 & 27 & 54 & 18 & 308 & 13 & 420 \\
4 & 40 & 13 & 294 & 66 & 7 & 420 \\
5 & 36 & 276 & 80 & 11 & 17 & 420 \\
Total & 420 & 420 & 420 & 420 & 420 & 2100
\end{tabular}

Source: Primary Data

TABLE 19: Percentage Position Determination

$\begin{array}{ccc}\text { Rank } & \text { Percentage }-100(\mathrm{Rij}-0.05) / \mathrm{Nj} & \text { Garret Score } \\ \mathbf{1} & 10 & \mathbf{7 6} \\ \mathbf{2} & 30 & \mathbf{6 1} \\ 3 & 50 & \mathbf{5 0} \\ 4 & \mathbf{7 0} & \mathbf{4 0} \\ \mathbf{5} & \mathbf{9 0} & \mathbf{2 5}\end{array}$

Source: Primary Data

TABLE 20: Garrett Mean Score

\begin{tabular}{|c|c|c|c|c|c|c|c|c|c|c|}
\hline \multirow{2}{*}{ Rank } & \multicolumn{2}{|r|}{ F1 } & \multicolumn{2}{|r|}{ F2 } & \multicolumn{2}{|r|}{ F3 } & \multicolumn{2}{|r|}{ F4 } & \multicolumn{2}{|c|}{ F5 } \\
\hline & Freq & GMS & Freq & GMS & Freq & GMS & Freq & GMS & Freq & GMS \\
\hline 1 & 11 & 1.990476 & 45 & 8.142857 & 24 & 4.342857 & 25 & 4.52381 & 315 & 57 \\
\hline 2 & 306 & 44.44286 & 32 & 4.647619 & 4 & 0.580952 & 10 & 1.452381 & 68 & 9.87619 \\
\hline 3 & 27 & 3.214286 & 54 & 6.428571 & 18 & 2.142857 & 308 & 36.66667 & 13 & 1.547619 \\
\hline 4 & 40 & 3.809524 & 13 & 1.238095 & 294 & 28 & 66 & 6.285714 & 7 & 0.666667 \\
\hline 5 & 36 & 2.142857 & 276 & 16.42857 & 80 & 4.761905 & 11 & 0.654762 & 17 & 1.011905 \\
\hline Total GMS & & 55.6 & & 36.88571 & & 39.82857 & & 49.58333 & & 70.10238 \\
\hline
\end{tabular}

Source: Primary Data

TABLE 21: Effectiveness factor to be improved on based on Garrett mean score

\begin{tabular}{|c|c|c|l|}
\hline Factor & Mean & Rank & \\
\hline F1 & 55.6 & 2 & Contractors to deliver their work/services as planned \\
\hline F2 & 36.8857143 & 5 & Inclusion and collaboration \\
\hline F3 & 39.8285714 & 4 & Transparency and accountability \\
\hline F4 & 49.5833333 & 3 & Community to fully own the project \\
\hline F5 & 70.102381 & 1 & Providing intended results \\
\hline
\end{tabular}




\section{SIX SET OF FACTORS}

TABLE 22: Efficiency Factor to be improved on

\begin{tabular}{|c||c||c||c||c||c||c||}
\hline S. No & F1 & F2 & F3 & F4 & F5 & Total \\
\hline \hline 1 & 0 & 100 & 20 & 70 & 230 & 420 \\
\hline \hline 2 & 21 & 0 & 30 & 230 & 139 & 420 \\
\hline \hline 3 & 30 & 220 & 70 & 69 & 31 & 420 \\
\hline \hline 5 & 268 & 30 & 101 & 21 & 0 & 420 \\
\hline \hline Total & 101 & 70 & 199 & 30 & 20 & 420 \\
\hline \hline
\end{tabular}

Source: Primary Data

TABLE 23: Percentage Position Determination

\begin{tabular}{ccc} 
Rank & Percentage $=\mathbf{1 0 0}(\mathbf{R i j - 0 . 0 5}) / \mathbf{N j}$ & Score \\
\hline 1 & 10 & 76 \\
2 & 30 & 51 \\
3 & 50 & 40 \\
4 & 70 & 25
\end{tabular}

Source: Primary Data

TABLE 24: Garret Mean Score

\begin{tabular}{|c|c|c|c|c|c|c|c|c|c|c|}
\hline \multirow{2}{*}{ S. No } & \multicolumn{2}{|r|}{ F1 } & \multicolumn{2}{|r|}{ F2 } & \multicolumn{2}{|c|}{ F3 } & \multicolumn{2}{|r|}{ F4 } & \multicolumn{2}{|c|}{ F5 } \\
\hline & Freq & GM Score & Freq & GM Score & Freq & GM Score & Freq & GM Score & Freq & GM Score \\
\hline 1 & 0 & 0 & 100 & 18.09524 & 20 & 3.619048 & 70 & 12.66667 & 230 & 41.61905 \\
\hline 2 & 21 & 3.05 & 0 & 0 & 30 & 4.357143 & 230 & 33.40476 & 139 & 20.1881 \\
\hline 3 & 30 & 3.571429 & 220 & 26.19048 & 70 & 8.333333 & 69 & 8.214286 & 31 & 3.690476 \\
\hline 4 & 268 & 25.52381 & 30 & 2.857143 & 101 & 9.619048 & 21 & 2 & 0 & 0 \\
\hline 5 & 101 & 6.011905 & 70 & 4.166667 & 199 & 11.84524 & 30 & 1.785714 & 20 & 1.190476 \\
\hline Total & Score & 38.15714 & & 51.30952 & & 37.77381 & & 58.07143 & & 66.6881 \\
\hline
\end{tabular}

Source: Primary Data

TABLE 25: Efficiency Factor to be improved on based on Garrett mean score

\begin{tabular}{|c|c|c|c|}
\hline Factor & Mean & Rank & \\
\hline F1 & 38.15714 & 4 & Timely provision of resources \\
\hline F2 & 51.30952 & 3 & Timely delivery of service and work \\
\hline F3 & 37.77381 & 5 & Funders to meet expectation \\
\hline F4 & 58.07143 & 2 & Standard Procurement procedures be fully implemented \\
\hline F5 & 66.6881 & 1 & $\begin{array}{l}\text { Council participatory budgeting structure be fully implemented and } \\
\text { independent }\end{array}$ \\
\hline
\end{tabular}




\section{RESULTS AND DISCUSSIONS}

The results from the research study, the respondents were able to identify and ranked the factors that needed serious improvements. These factors the respondents found out were: 1 . Availability of funds; 2 . empowerment of citizens; 3. Strong implementation, monitoring, evaluation and reporting; 4. their ward to benefit from participatory budgeting projects; 5. councils and other ministries, departments and agencies (MDAs) to be highly inclusive and collaborative and; 6 . recommended that funders to meet their expectations. If the above factors are improved on, the effectiveness and efficiency of participatory budgeting in the Port Loko District and City council will achieve community development goals.

\section{CONCLUSION}

The recommendations of improvement factors for effective and efficient Participatory budgeting in the Port Loko District and City Councils to achieve future programme implementation that is fully participatory in nature; the research from the respondents recommends that, there should be available funds, the councils to empower their citizens, there should be strong implementation, monitoring, evaluation and reporting of all participatory budgeting activities, they all want their ward to benefit from Participatory budgeting projects, they further recommended that councils and other ministries, departments and agencies (MDAs) to highly include and collaborate with many stakeholders in their sovereignty if they want the project to be highly effective and efficient in the future and lastly as money is the back bone to every project they want funders to meet their expectations in relation to time of release of funds. When the above improvements are done there will be effective and efficient participatory budgeting process that will contribute to high community development in their localities.

\section{REFERENCES}

[1] ACT, L. G. (2004, 03 1). THE LOCAL GOVERNMENT ACT, 2004. Retrieved 1101,2020 , from The Local Government Act, 2004-Sierra Leone Web: http://www.sierra-leone.org/Laws/2004-1p.pdf.

[2] Commonwealth Governance (n.d.). Local Government. Retrieved 06 07, 2021, from Local Government of Sierra Leone

https:/www.commonwealthgovernance.org/ countries/africa/sierra_leone/local-government/.

[3] EUKN.(n.d.). Participatory Budgeting ? Retrieved 11 1, 2020, from European Urban Knowledge Network: https:/www.eukn.eu/policy-labs/policy-lab-for-cypublic-participation-in-the-developmentprocess/participatory-budgeting/.

[4] LGA. (2020). Participatory Budgeting. Retrieved 11 1, 2020, from Local Government Association: https:/www.local.gov.uk/topics/devolution/engagingcitizens-devolution/approaches-civic-anddemocratic-engagement- 0 .

[5] OCHA Services. (2016, 01 04). Sierra Leone: Port Loko District Profile. Retrieved 06 07, 2021, from reliefweb:https:/reliefweb.int/report/sierraleone/sierra-leone-port-loko-district-profile-29. december-2015.

[6] Rahman, S. Z.-u. (2011, 01 24). Henry Garrett Ranking Techniques. Retrieved 06 06, 2021, from Scribd: https://www.scribd.com/doc/47487605/HenryGarrett-Ranking-Techniques. 